\title{
Bond Graph Modeling, Analysis and Control of Dual Stage System
}

\author{
Wei-Jun Wang ${ }^{1}$ and Chang-Soo Han $^{1^{*}}$ \\ ${ }^{1}$ Mechanical Engineering, Hanyang University \\ 본드그래프를 이용한 듀얼 스테이지 시스템의 모델링, \\ 해석, 및 제어 \\ 왕위준 ${ }^{1}$, 한창수 ${ }^{*}$ \\ ${ }^{1}$ 한양대학교 기계공학과
}

\begin{abstract}
The dual stage manipulator is composed of the voice coil motor (VCM) and piezoelectric ceramics transducer (PZT), which can produce the high precise displacement and express a well dynamic performance. However, inaccurate modeling of the dual stage will exacerbate the positioning accuracy. This paper presents an approach to model the dual stage system by using bond graph theory. And the state space equations can be derived through the bond graph straightforwardly, which can be used in computing simulations. Through designing the compensators for the dual stage system and simulating, the dual stage performs better dynamics characteristic than the single actuator system.
\end{abstract}

요 약 본 연구에서는 보이스코일 모터와 압전 구동기를 동일 축으로 배치함으로써 초정밀 위치결정뿐만 아니라 동 특성을 개선할 수 있도록 하는 듀얼 스테이지를 다룬다. 듀얼 스테이지는 모델링이 엉성할 경우 오히려 나쁜 동특성 을 보이는 단점이 있다. 이 논문은 본드그래프를 이용하여 듀얼 스테이지를 모델링하고 동적 상태방정식을 본드그래 프에서 유도하였다. 또한 동특성을 향상시키기 위한 보상기의 설계 예시를 보이고 있으며, 시뮬레이션을 통해 검증하 였다.

Key Words : Dual stage, Bond graph, Modeling and simulation, Control

\section{Nomenclature}

symbol Description

$U_{i v} \quad$ Input voltage for $\mathrm{VCM}$

$i_{v} \quad$ Equivalent current for VCM

$L_{v} \quad$ Equivalent inductance for VCM

$B_{v c m} \quad$ VCM equivalent damping

$M_{c s} \quad$ Equivalent mass of VCM mover and coarse stage

$K_{v c m} \quad$ Sum of VCM servo stiffness and spring stiffness
$F_{v c m} \quad$ VCM driver force

$X_{v c m} \quad$ Displacement of coarse stage

$V_{v \mathrm{~cm}} \quad$ Velocity of coarse stage

$U_{i p} \quad$ Input voltage for PZT

$B_{p z t} \quad$ PZT equivalent damping

$M_{f s} \quad$ Equivalent mass of fine stage

$F_{p z t} \quad$ PZT driver force

$X_{p z t} \quad$ Displacement of fine stage

$V_{p z t} \quad$ Velocity of fine stage

This research was supported by the MKE(The Ministry of Knowledge Economy), Korea, under the Advanced Robot Manipulation Research Center support program supervised by the NIPA(National IT Industry Promotion Agency)" (NIPA-2011-C7000-1001-0002)

*Corresponding Author : Han, Chang-Soo

Tel: +82-10-8777-5247 e-mail: cshan@hanyang.ac.kr

Received March 2, 2012

Revised March 16, 2012

Accepted April 12, 2012 


\section{Introduction}

Dual stage mechanism is a complicated system which embodies concepts from the mechanical, electrical and magnetic domains. Various examples for the concept of dual stage mechanism have been developed in the past. The successful application of dual stage concept was the data storage systems such as hard disk drives, where the VCM drives the fine actuator, PZT, which is mounted between the suspension and the base plate [1]. In the paper [2], the linear dual stage consisting of two VCMs were used to transfer the force at the end point of the whole stage, which was used as chip-on-board assembly. And there were some applications of dual stage as the high-speed/high precision positioning system for the IC/MEMS manufacturing, high speed precision machining [3].

Before the real system is designed, the most important attempt must begin with a prediction of its performance in advance. There are several approaches to build the system model to describe its performance. One approach is mathematical equation described in terms of the differential equations, which is a very universal method for single-field physical systems, and it is easy to analyze by using software. A much greater number of research papers have been adopted at this method[4]. Another approach is graphic method, such as block diagram, signal flow graph, bond graph and so on. One can derive the mathematical model of the actual system from previously mentioned methods. Even though block diagram and signal flow graph describe the system as a picture of the equation, they do not discern the topology of the system profoundly. Bond graph theory is based on energy and information flow, which is a modeling and analysis technique in multi-field systems, invented in 1959 by Paynter. It is publicly known to be highly appropriate powerful tool for dynamic modeling after it comes out. And another merit is it enables one to develop a graphical model that is consistent with the principle of energy conservation without establishing and reformulating equations. This paper focuses on the use of bond graph method for modeling and simulating the dual stage system. The real dual linear motion stage with single axis can be represented as Fig. 1.

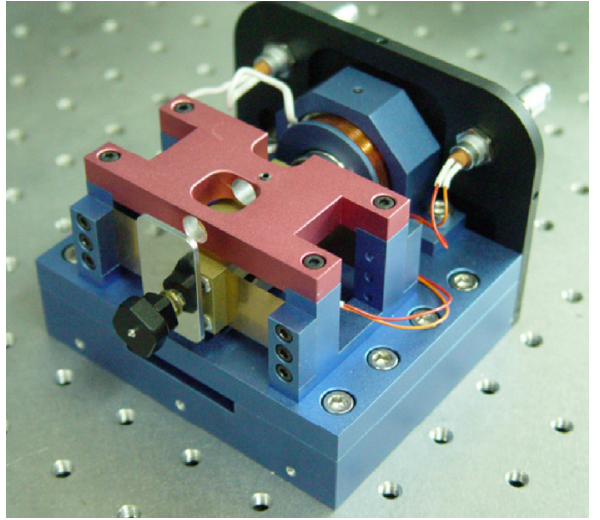

[Fig. 1] Dual stage composed of VCM and PZT

The rest paper is organized as follows. Section 2 describes the approach of building the bond graph models of the dual stage mechanism, while section 3 shows how to obtain the state space equation from the bond graph models and obtain the simulation result. And section 4 describes the controller design methods and simulation results based on the controller. Finally, section 5 contains concluding remark.

\section{Bond Graph Modeling}

The dual stage system is composed of two parts, the coarse stage and the fine stage which shown in Fig. 2. The coarse stage is collected with the moving magnet of the VCM and supported by the four leaf springs, which is driven by the VCM and produce long distance. Furthermore, the PZT is fixed with the coarse stage rigidly, and the fine stage which is very light, is mounted at the endpoint of the PZT. For describing the dynamic behaviors accurately, the coarse and fine stages are separated to analyze firstly. Finally, the bond graph of the whole system are derived.

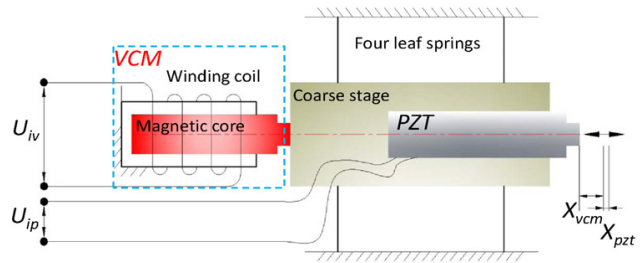

[Fig. 2] Schematic structure diagram of dual stage 


\subsection{Bond Graph Modeling of the Coarse Stage}

From the working principle of the VCM, a permanent magnetic core can move inside winding coil which is fixed on the base stage after inputting the current to the winding coil. With the change of the current quantity or the current direction, the permanent magnetic core will move back and forth. The dynamic model of the VCM-based electromagnetism and mechanism can be seen in Fig. 3.

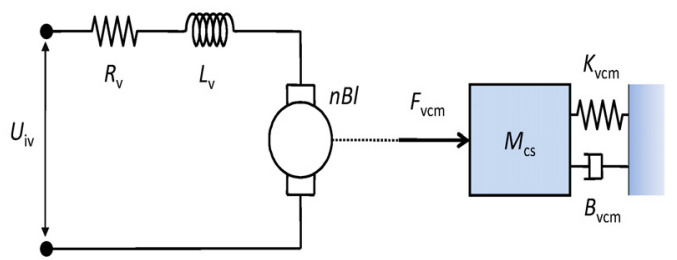

[Fig. 3] Dynamic model of the VCM-based electromagnetism and mechanism element

The mathematic equation for this mechanism can be described as (1).

$$
\begin{aligned}
& \left(U_{i v}-\frac{d}{d t}\left(L_{v} i_{v}\right)-R_{v} i_{v}\right) i_{v} \\
& =\left(\frac{d}{d t}\left(M_{c s} V_{v c m}\right)+B_{v c m} V_{v c m}+K_{v c m} \int V_{v c m} d t\right) V_{v c m}
\end{aligned}
$$

From the Equation 1, it can be seen that each term of equation is having the power unit. The three terms on the left-hand side are the power equivalent which acts as the electric domains and the magnetic domains respectively. The three terms on the right side are the power equivalent which acts as the mechanical domains, representing the potential power, kinetic power and dissipative power respectively. The left element of every term represents the flow quantities, and the right element of every term is the effort quantities. From the former analysis and Equation 1 , the bond graph of the coarse stage is derived and described in Fig. 4.

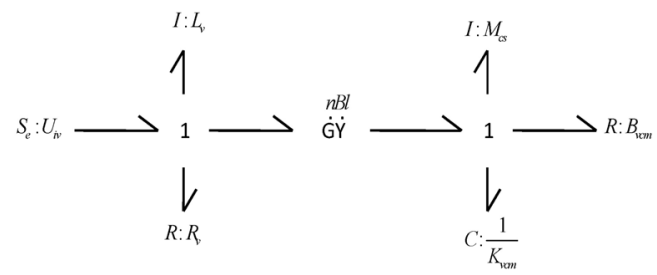

[Fig. 4] Bond graph model of coarse stage

\subsection{Bond Graph Modeling of the Fine Stage}

The purpose of using fine stage is to adjust the coarse stage positioning and displacement, and to add the pronounce effect to the resolution of the movements. The dynamic model of the PZT-based mechanism is shown as Fig. 5. [5]

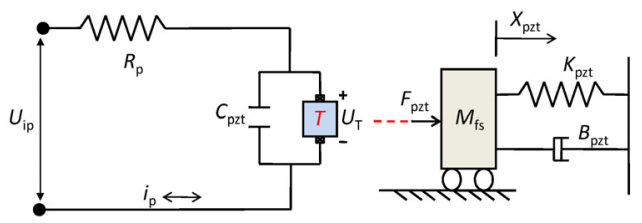

[Fig. 5] Dynamic model of PZT-based mechanism

From the Fig. 5, the model of the PZT is composed of mechanical field and electrical field. At the electrical field, $R_{p}$ is the equivalent resistance of driving circuit, and a stack designed piezoelectric ceramics actuator is made up of several wafer elements, each wafer element is located between positive electrode and negative electrode, which is expressed as characteristic of capacitance element. $C_{p z t}$ is the equivalent capacitance of each wafer in parallel electrically. $U_{i p}$ and $U_{T}$ are input voltage and the voltage across the capacitor, respectively.

The mathematic equation for this mechanism can be described as (2) and (3) in the mechanical part and electrical part, respectively.

$$
\begin{aligned}
& F_{p z t}=\frac{d}{d t}\left(M_{f s} V_{p z t}\right)+B_{p z t} V_{p z t}+\int K_{p z t} V_{p z t} d t \\
& U_{i p}-\left(\frac{d}{d t}\left(C_{p z t} U_{p z t}+T V_{p z t}\right)\right) R_{p}=U_{p z t}
\end{aligned}
$$

From the above two equations, the bond graph of the fine stage is derived and described as Fig. 6. 


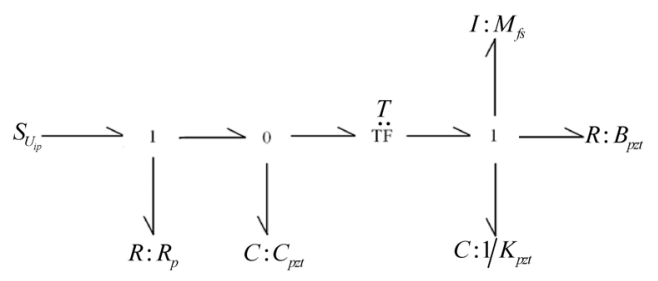

[Fig. 6] Bond graph model of fine stage

\subsection{Bond Graph Modeling of the Whole Stage}

From the foregoing statement, the whole stage have two inputs and one output, and the fine stage is fixed to the coarse stage, then the mutual stiffness of two stages is so high that it can be considered two stages as one rigid body. On the contrary, the mutual damping of two stages is so small that it can be considered there is no damping of those two stages. However, the mass of fine stage is much smaller than the coarse stage, which means the interference of the coarse stage to the fine stage is higher than the opposite case. In order to eliminate the interference of each other, it is possible to compensate for the loss of fine stage by using the control algorithm, or build precise model to eliminate the interference. The whole stage bond graph is described as Fig. 7.

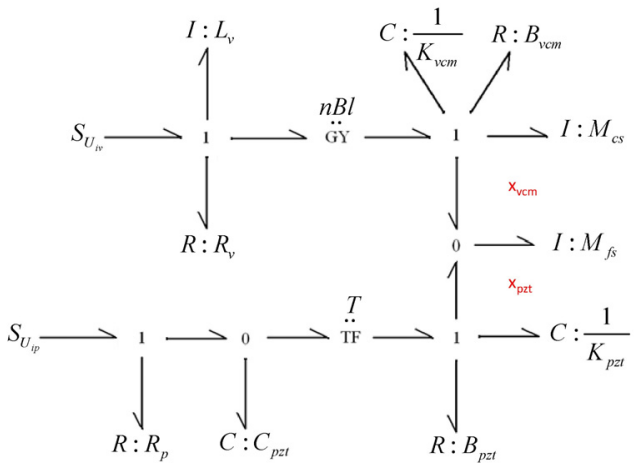

[Fig. 7] Bond graph model of whole stage

From the bond graph, it is clear to see the displacement from coarse system was $X_{p z t}$, and from fine system was $X_{p z t}$, so the displacement of the dual stage was embodied by the mass of the fine stage, $M_{f s}$.

\section{State space equation and simulation}

The purpose to derive the bond graph of system is to analyze the system, and the enthusiasm is to simulate the system through bond graph conveniently. At present, there are several commercial software programs which symbolic and numeric solution capabilities are very advanced, such as CAMP-G, 20-SIM, etc. In this paper, by deriving system equations and obtaining computer solutions from these equations, it is easy to detect the predominance for constructing bond graph models apparently.

\subsection{State Space Equation of the Whole System}

Before deriving the system state space equation, there are two essential processes to improve the bond graph, one is to number all the bonds in continuous order, and another is the assignment of causality. it is always bringing the generalized momentum $p$ of inertia element and the generalized displacement $q$ of capacitance element into the state variable of system [6]. For the dual stage, there are 3 state variables in the coarse stage, 3 state variables in the fine stage, and 6 state variables in the whole stage. The modified bond graph of whole system is shown as Fig. 8.

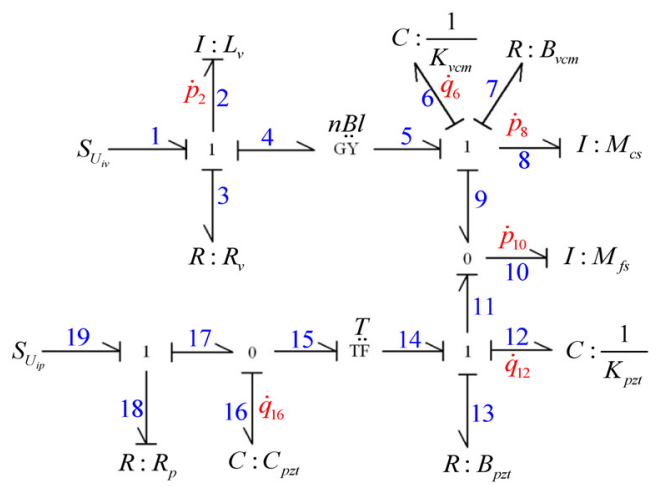

[Fig. 8] Augmentation of the bond graph model

Now by using the fully integration causality relationship of bond graph, it is easy to derive the state space equations of the whole system. The state space equations are showed at Appendix. 


\subsection{Simulation Result of the Whole System}

Step response and frequency response of the opened-loop system are used to simulate the whole stage. The parameters of the coarse stage and fine stage used in the simulation are listed as Table 1. The step response of the whole stage is described as Fig. 9.

[Table 1] Parameters of coarse and fine stage used in simulation

\begin{tabular}{|l|l|l|}
\hline \multicolumn{1}{|c|}{ parameter } & \multicolumn{1}{c|}{ Symbol } & \multicolumn{1}{c|}{ value } \\
\hline Mass of coarse & $M_{c s}$ & $0.095 \mathrm{~kg}$ \\
\hline Stiffness of coarse & $K_{v c m}$ & $3014 \mathrm{~N} / \mathrm{m}$ \\
\hline Damping of coarse & $B_{v c m}$ & $4.9 \mathrm{Ns} / \mathrm{m}$ \\
\hline Transformer of coarse & $n B l$ & $\begin{array}{l}N=45, \\
B=0.2 \mathrm{~T} \\
l=0.091 \mathrm{~m}\end{array}$ \\
\hline Coil resistance & $R_{v}$ & $0.98 \Omega$ \\
\hline Coil inductance & $L_{v}$ & $6.326 \times 10^{-5} \mathrm{H}$ \\
\hline Mass of fine & $M_{f s}$ & $0.02 \mathrm{~kg}$ \\
\hline Stiffness of fine & $K_{p z t}$ & $5.7 \times 10^{6} \mathrm{~N} / \mathrm{m}$ \\
\hline Damping of fine & $B_{p z t}$ & $150 \mathrm{~ns} / \mathrm{m}$ \\
\hline Capacitance of fine & $C_{p}$ & $1.5 \times 10^{-6} \mathrm{~F}$ \\
\hline Transformer ratio & $T$ & $12.5 \mathrm{c} / \mathrm{m}$ \\
\hline Circuit resistance & $R_{p}$ & $0.9 \Omega$ \\
\hline
\end{tabular}
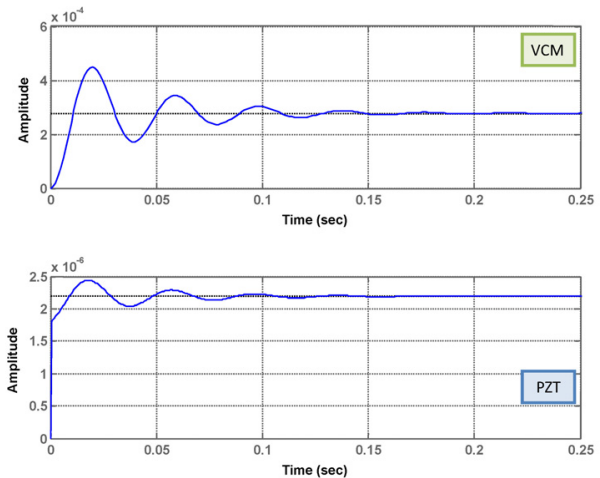

[Fig. 9] Step response of the whole stage for different parts

From Fig. 9, there are two response curves, one curve is the displacement output comes from the VCM, and another curve is the displacement output comes from the PZT. Both of them can achieve the stabilization quickly, which means the modeling and the parameters for the real stage is reasonable. But the overshoot of the coarse stage is high and the settling time for both stages is long for precision system, it needs to design the controller to change those time properties.

\section{Controller and simulation}

Dual system is a double-input single-output(DISO) system, which is a special case of a multi-input multi-output (MIMO) system. In such circumstances, some authors used single-input single-output (SISO) frequency technique/tools to design the DISO controllers, such as Master-Slave method [7], PQ method [8]. These three methods use the perfect SISO tools to design the controller based on simplifying the system architecture of the dual stage. In the paper, PQ method will be used to design the controller.

There are some merits for PQ method. It is specialized to DISO plants, it simplify the DISO design problems to two consecutive SISO designs, especially overcome the interference problems of two actuating stage which Master-Slave method cannot avoid. And another very important merit is the order of compensator which design by using PQ method is low but effective. Fig. 10 shows the block diagram of single-input double-output (SIMO) controller for MISO plant [8].

By using the PQ method, Fig. 11 shows that the dual stage with controller can attain well dynamic performance.

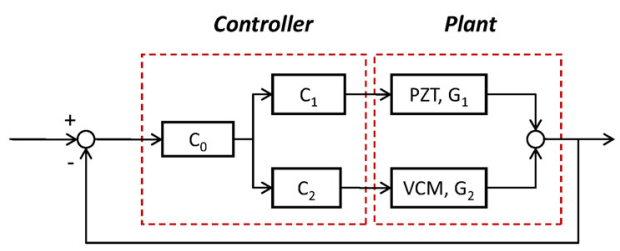

[Fig. 10] Block diagram of DISO system with a single-input double-output (SIDO) compensator

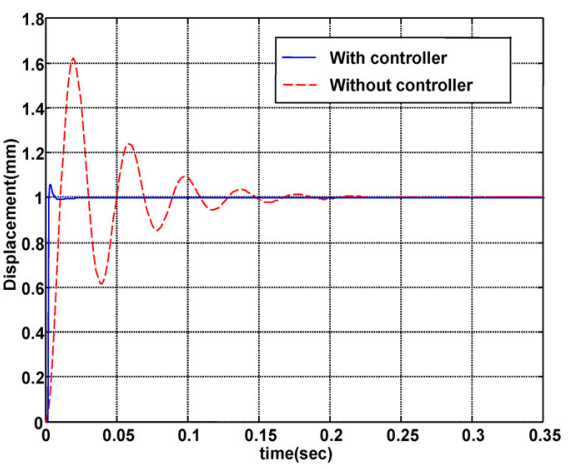

[Fig. 11] Step response of with controlled system and without controlled system 


\section{Conclusions}

The main contribution of this paper is to use bond graph theory to model the dual stage system. Building the precise and reasonable model is the precondition to design the control system by using the language of the bond graph. In this paper, all state variables are considered which is belonged to the mechanical and electrical domain. Also from the simulation results, it is easy to draw a conclusion that the model of dual stage can reach a steady step response. However, from the step response, it can't perceive the dynamic performance of the dual stage system because of its open-loop system. But after using PQ method to design the controller, and analyzing the simulation results, it is clearly to see that the dual stage performed better than the single subsystem.

\section{Appendix}

Dual stage state-space equation

$\dot{x}=A x+B u \quad y=C x$

State-space variables, input and output variables

$x=\left[\begin{array}{llll}p_{2} & q_{6} p_{8} p_{10} q_{12} q_{16}\end{array}\right]^{T}, u=\left[\begin{array}{ll}U_{i v} & U_{i p}\end{array}\right]^{T}$

Coefficient matrices

$$
\begin{aligned}
& A=\left[\begin{array}{cccccc}
-\frac{R_{v}}{L_{v}} & 0 & -\frac{n B l}{M_{c s}} & 0 & 0 & 0 \\
0 & 0 & \frac{1}{M_{c s}} & 0 & 0 & 0 \\
\frac{n B l}{L_{v}}-K_{v c m}-\frac{B_{v c m}+B_{p z t}}{M_{c s}} & \frac{B_{p z t}}{M_{f s}} & K_{p z t} & -\frac{T}{C_{p z t}} \\
0 & 0 & \frac{B_{p z t}}{M_{c s}} & -\frac{B_{p z t}}{M_{f s}}-K_{p z t} & \frac{T}{C_{p z t}} \\
0 & 0 & -\frac{1}{M_{c s}} & \frac{1}{M_{f s}} & 0 & 0 \\
0 & 0 & \frac{T}{M_{c s}} & -\frac{T}{M_{f s}} & 0 & -\frac{1}{R_{p} C_{p z t}}
\end{array}\right], B=\left[\begin{array}{ll}
1 & 0 \\
0 & 0 \\
0 & 0 \\
0 & 0 \\
0 & 0 \\
0 & \frac{1}{R_{p}}
\end{array}\right] \\
& C=\left[\begin{array}{lllll}
0 & 10 & 0 & 10 & 0
\end{array}\right]
\end{aligned}
$$

\section{References}

[1] S.M. Suh, C.C. Chung, S.H. Lee, "Design and analysis of dual-stage servo system for high track density HDDs", Microsystem Technologies, Vol. 8, 2002, pp. 161-168.

[2] Norbert C. Cheung and Byron M.Y. Cheung, "Modelling and control of a High Speed, Long Travel, Dual Voice Coil Actuator," IEEE International Conference on
Power Electronics and Drive Systems, Vol. 1, pp. 270-274. 2002.

[3] Jie Degang, "Research on the High-Speed/High-Precision Positioning System of Macro/Micro Driving," Ph.D thesis, Harbin Institute of Technology, 2006.

[4] Tielong Shen and Minyue Fu, "High Precision Feedback Control Design for Dual-Actuator Systems," IEEE Conference on Cotrol Applications, Canada, August, 2005.

[5] Michael Goldfard and Nikola Celanovic, "Modeling Piezoelectric Stack Actuators for Control of Micromanipulation," IEEE International Conference on Robotics and Automation, Minneapolis, MN, April 1996.

[6] Dean C. Karnopp, Donald L. Margolis, System Dynamics-Modeling and Simulation of Mechatronic Systems, 4th ed., John Wiley \& Sons, Inc. 2005, pp. 155-193.

[7] S.Koganezawa, Y. Uematsu, T. Yamada, "Dual-stage actuator systems for magnetic disk drives using a shear mode piezo-electric microactuator", IEEE Transactions on Magnetics, 35(2), 1999, pp. 988-991.

[8] S.J.Schroeck, W.C.Messner, "On compensator design for linear time-invariant dual-input single-output system", IEEE/ASME Transactions on mechatronics, 6(1), March 2001, pp. 50-57.

\section{Wei-Jun Wang}

[Regular member]

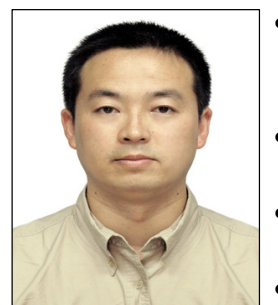

- Jul. 1999 : Xiaan University of Architechure \& Technology, China, BS

- Sept. 1999 Jul. 2003, Wuhan Iron and Steel Corp., Engineer

- Jul. 2006 : China University of Geogsciences., China, MS

- Sept. $2008 \sim$ current : Hanyang Univ., PHD Candidate

$<$ Research Interests $>$

Hybrid control system, parallel robots and ultra precision mechanism based flexure hinge 


\section{Chang-Soo Han}

[Regular member]

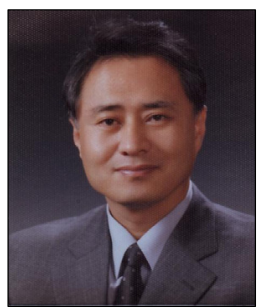

- 1985 : The University of Texas at Austin, USA, MS

- 1989 : The University of Texas at Austin, USA, PhD

- Mar. $1990 \sim$ current : Hanyang Univ., Dept. of Mechanical engineering, Professor

$<$ Research Interests $>$

High precision robotics and mechatronics, rehabilitation and biomechanics technology using robotics, automation in construction, and advanced vehicle control 